\title{
Pertinence du concept de 'borne continue' dans une théorisation des notions aspectuo-temporelles
}

\author{
Jean-Pierre Desclés ${ }^{11}$, et Zlatka Guentchéva ${ }^{2}$ \\ ${ }^{1}$ Sorbonne Université, STIH, 28, rue Serpente, 75006 Paris \\ ${ }^{2}$ LACITO-CNRS, 7, rue Guy Môquet (Bât.D)-94801 Villejuif Cedex
}

\begin{abstract}
Résumé. L'aspect grammatical est un opérateur qui actualise une relation prédicative (ou dictum) sur un intervalle topologique d'instants contigus dans un référentiel temporel (continu). L'intervalle est ouvert avec un état, fermé avec un événement et semi-ouvert (à droite) avec un processus. La notion de borne (ouverte ou fermée) joue un rôle important dans la théorisation et la description des oppositions sémantiques entre les principales valeurs aspectuo-temporelles. Lorsque la temporalité est continue, la borne qui sépare un événement et un état résultant est nécessairement une coupure continue : elle est le dernier instant de l'événement mais elle n'est pas le premier instant de l'état résultant, c'est une borne fermée de l'événement et en même temps une borne ouverte de l'état résultant. L'acte d'énonciation construit un référentiel énonciatif autonome, déconnecté du référentiel externe. L'analyse des discours rend nécessaire la prise en compte de différents référentiels (narratif, hypothétique, discours rapportés...). L'article se propose de clarifier ces conceptualisations.
\end{abstract}

\begin{abstract}
Relevance of the concept of continuous boundary in the theorization of aspecto-temporal notions. Grammatical aspect is an operator that actualizes a predicative relation (or dictum) onto a topological interval of contiguous instants inside of a temporal (continuous) frame. The notion of (open or closed) boundary plays an important role in the theorization and the description of different aspectuo-temporal values. With a state, the interval is open, it is closed with an event and semi-open (at right) with a process. When temporality is continuous, the boundary between an event and a resulting state is necessarily a continuous cut: it is the last moment of the event and closed in the actualization of an event but it is not the first moment of the resulting state, it is an open boundary. The act of speaking constructs an autonomous enunciative frame that is disconnected from the external frame. The discourse analysis necessitates to articulate different temporal frames (narrative, hypothetic, reported speeches ...). The aim of this article is clarification of these abstract conceptualizations.
\end{abstract}

Les notions d'intervalle et de borne, ainsi que celles de bornage, de borné ou non borné, sont souvent utilisées pour décrire les phénomènes aspectuo-temporels à travers la diversité des langues mais ne reçoivent pas toujours une interprétation univoque. Dans l'analyse des temps grammaticaux de H. Reichenbach (1947), par exemple, la notion de borne n'est pas pertinente puisque l'auteur ne recourt pas à des intervalles mais uniquement à des instants. L. Gosselin $(1995,2010)$ complexifie les analyses et représentations de Reichenbach en introduisant des intervalles d'instants sans toutefois exploiter les propriétés topologiques des différents types de borne, notamment lorsque l'on considère une continuité temporelle sous-jacente. Par ailleurs, malgré une abondante littérature consacrée à l'aspectualité, on constate une confusion dans l'opposition borné / non borné, la notion de 'non-borné' étant généralement interprétée comme " une absence de bornes » appartenant à un intervalle. Or, des énoncés comme Pendant des milliers d'années, les hommes étaient uniquement des chasseurs-cueilleurs ou Ce jour-là, l'après-midi était chaude expriment clairement des états qui sont bornés puisqu'une durée mesure la distance entre les deux bornes de ces

\footnotetext{
${ }^{1}$ jeanpierre.descles@gmail.com
} 
états ; il n'y a donc pas une absence de bornes mais les deux bornes de chaque état n'appartiennent pas aux intervalles d'actualisation de ces états. L'analyse sémantique se doit d'éviter de recourir à des oppositions notionnelles surchargées. A ce propos, rappelons le conseil épistémologique de Gaston Bachelard :

\begin{abstract}
On devrait toujours se méfier d'un concept qu'on a pas encore su dialectiser. Ce qui empêche sa dialectisation, c'est une surcharge de son contenu. Cette surcharge empêche le concept d'être délicatement sensible à toutes les variations des conditions où il prend ses jutes fonctions. A ce concept on donne sûrement trop de sens puisque jamais on ne le pense formellement. Mais si on lui donne trop de sens, il est à craindre que deux esprits différents ne lui donnent pas le même sens.

(Bachelard 1940/1966: 134)
\end{abstract}

Depuis Galilée, l'histoire des sciences nous a montré que l'effort de mathématisation des concepts d'une discipline (comme l'étude du mouvement et du changement) a toujours contribué à faire progresser considérablement sa théorisation, à en affiner les descriptions et à faire surgir des interrogations plus profondes. Dans la mesure où, dans l'étude sémantique des catégories grammaticales, comme celles du temps, de l'aspect et des modalités (TAM), les linguistes recourent souvent aux notions d'intervalle, d'instant et de borne, il paraît naturel qu'ils cherchent à reprendre les définitions opératoires et précises de ces concepts que la mathématique a progressivement élaborés avec la topologie générale. Le présent article vise à préciser les notions topologiques d'instant, de continuité temporelle, de borne temporelle et d'intervalle ouvert ou fermé, employées, souvent sans définition, dans leurs représentations métalinguistiques de la catégorie du TAM. La représentation à l'aide de diagrammes théorisés est souvent un support intuitif utile pour caractériser (en le dialectisant) plus exactement ces concepts et les opposer à des concepts proches.

\title{
1 Concept de 'borne continue'
}

Les situations référentielles auxquelles renvoient les énoncés sont actualisées sur des intervalles d'instants contigus. La temporalité sous-jacente à ces actualisations (aussi bien en physique classique qu'en linguistique) est plutôt continue que discrète, comme le défend le mathématicien René Thom :

Selon le point de vue traditionnel, le continu se construit par complétion à partir du dénombrable (...). Or moi, je pense l'inverse : c'est l'infini dénombrable qui est justifié par son immersion dans le continu. (...) Je suis convaincu que les structures les plus discrètes sortent d'une espèce de génération continue sous-jacente.

(Thom 1983: 147)

La notion cognitive de continuité doit être considérée comme première par rapport à la notion de dénombrabilité discrète (contrairement aux constructions axiomatiques de la théorie des ensembles). Le caractère discontinu exprimé par des énoncés comme $I l$ prend tous les jours ses repas à midi trente ou Il se promène le soir, après le dîner, pendant une heure renvoie à des habitudes qui sont actualisées sur un certain nombre d'intervalles qui se détachent d'un support temporel continu.

Comment caractériser la continuité d'un référentiel temporel ? La continuité est souvent confondue avec la densité qui est caractérisée par la propriété suivante : " entre deux instants, il existe toujours un instant intermédiaire ». Or, la compréhension de la nature de l'ensemble totalement ordonné des nombres réels, mis en correspondance avec la ligne temporelle des instants, a conduit, à la fin du $\mathrm{XIX}^{\circ}$ siècle, des mathématiciens comme A.-L. Cauchy, R. Dedekind, G. Cantor, à caractériser la continuité, en la distinguant de la densité, pour tenir compte du fait qu'un nombre comme $\sqrt{2}$ peut être seulement approximé par des suites de nombres fractionnaires $\mathrm{p}_{\mathrm{i}} / \mathrm{q}_{\mathrm{i}}$ sans lui-même être un rapport rationnel de la forme $\mathrm{p} / \mathrm{q}$, tout en étant un « vrai nombre réel ». R. Dedekind $(1888)^{\mathrm{i}}$ a précisé la nature continue de tous les nombres réels en faisant appel au concept de « coupure continue ». Précisons brièvement ce concept abstrait. Dans le parcours de la ligne temporelle totalement ordonnée des instants consécutifs (ou des points successifs d'une droite orientée), une coupure est un instant (ou un point) qui détermine deux domaines temporels complémentaires : le domaine $\mathbf{A}_{1}$ de tous les instants qui précèdent la coupure et le domaine $\mathbf{A}_{2}$ de tous les instants qui sont postérieurs à la coupure ; cette coupure est définie comme continue lorsqu'elle appartient soit exclusivement à $\mathbf{A}_{1}$, soit exclusivement à $\mathbf{A}_{2}$ mais jamais à leur intersection (vide, par définition du concept de coupure); cela revient à dire que, entre deux instants (ou 
points) consécutifs quelconques de part et d'autre de la coupure continue, il n'y a aucune place ni pour un «saut » qui indiquerait alors une absence d'instants intermédiaires entre ces deux instants consécutifs, ni pour une « lacune », ce qui impliquerait alors l'existence d'un éventuel instant intermédiaire, ce dernier n'étant pas atteint par le parcours continu de tous les instants consécutifs de $\mathbf{A}_{1}$ puis de $\mathbf{A}_{2}$. Dans le parcours continu de $\mathbf{A}_{\mathbf{1}} \cup \mathbf{A}_{2}$, la coupure appartient soit à $\mathbf{A}_{1}$ constituant ainsi une borne fermée (le dernier instant du parcours de $\mathbf{A}_{1}$ ) tout en constituant une borne ouverte à la gauche de $\mathbf{A}_{2}$ mais n'appartenant pas à $\mathbf{A}_{2}$, soit à $\mathbf{A}_{2}$, constituant ainsi une borne fermée (le premier instant du parcours de A2) tout en constituant une borne ouverte de $\mathbf{A}_{1}$ sans toutefois y appartenir (Figure 1).

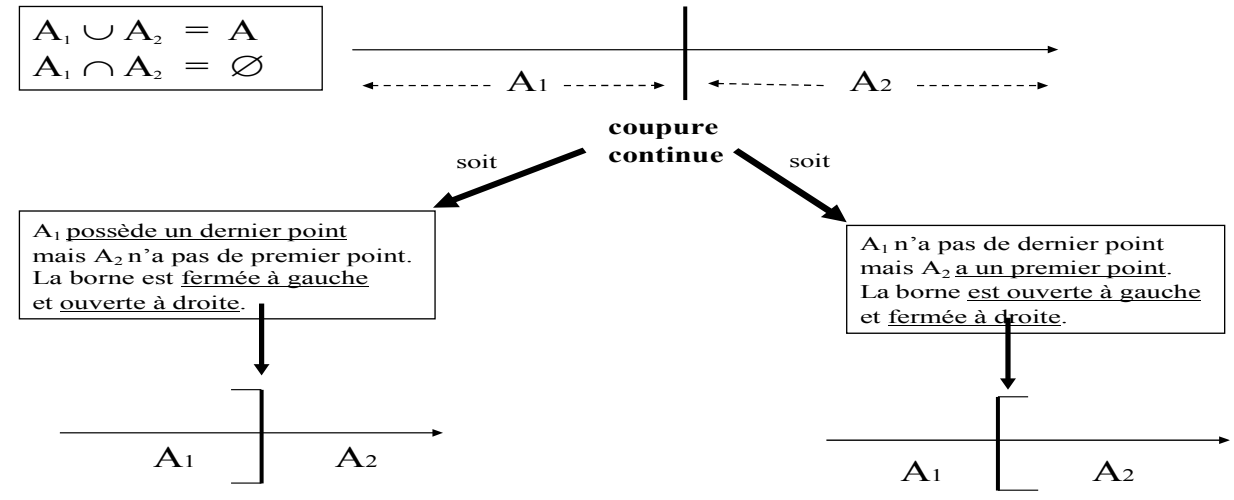

Figure 1: Coupure continue (Dedekind)

Nous pouvons ainsi concevoir différents types d'intervalles bornés sur lesquels sont actualisées des situations. Une borne d'un intervalle 'I' est ouverte lorsque qu'elle n'appartient pas à l'intervalle 'I', bien qu'elle puisse être approchée, aussi près que l'on veut, par des instants de l'intervalle 'I'. Une borne d'un intervalle 'I' est fermée lorsqu'elle appartient nécessairement à l'intervalle 'I'. Un intervalle borné d'instants est dit ouvert, respectivement fermé, lorsque ses deux bornes (à gauche et à droite) sont ouvertes, respectivement fermées ; un intervalle est semi ouvert (à droite) lorsqu'il possède une borne ouverte à droite et une borne fermée à gauche. Selon nous, ces concepts topologiques sont essentiels pour préciser la sémantique de l'aspectualité, notamment pour formaliser les concepts d'inaccompli, d'accompli, d'achevé (c'est-à-dire de perfectivité), d'état résultant...

\section{Aspectualisation de base : état, événement et processus}

De nombreuses approches consacrées à l'aspectualité ne retiennent que l'opposition aspectuelle binaire état $v s$ événement et ignorent la notion de processus, ce qui conduit souvent à des imprécisions terminologiques en confondant, par exemple, activité et ce qui devrait être interprété comme processus. ${ }^{\text {ii }}$ Pourtant, d'excellents linguistes, comme B. Comrie (1976), J. Lyons (1977), A. Mourelatos (1981), ont explicitement recours à la notion de processus (sans en donner toutefois une définition opératoire) qu'ils opposent à celles d'état et d'événement. Le concept de processus nous paraît indispensable pour entreprendre une description adéquate et économique des principales valeurs aspectuelles grammaticalisées par les langues, comme le remarque aussi H.-J. Sasse (2002).

Les aspects de base 'état', 'événement' et 'processus' sont analysés et formalisés sous la forme d'opérateurs appliqués à une relation prédicative (ou dictum), ce qui revient à aspectualiser la situation (c'est-à-dire la dénotation de la relation prédicative) en précisant comment elle est actualisée sur un certain type d'intervalle caractérisé par des bornes topologiques ouvertes ou fermées. ${ }^{\text {iii }}$ 
Une relation prédicative (ou dictum) aspectualisée sous la forme d'un état est actualisée sur un intervalle ouvert ' $\mathrm{O}$ ' (avec les bornes ouvertes à gauche et à droite de ' $\mathrm{O}$ ', donc exclues de ' $\mathrm{O}$ '), au moyen de l'opérateur 'ETAT ${ }_{O}$ ' qui est appliqué sur elle. Une relation prédicative aspectualisée sous la forme d'un événement est actualisée sur un intervalle fermé ' $\mathrm{F}$ ' (avec les bornes fermées à gauche et à droites de ' $\mathrm{F}$ ', donc appartenant à ' $\mathrm{F}$ '), d'où l'opérateur 'EVEN ${ }_{\mathrm{F}}$ ' appliqué sur elle. Une relation prédicative aspectualisée sous la forme d'un processus s'actualise sur un intervalle semi-ouvert ' $J$ 'dont la borne initiale à gauche est fermée (indiquant le début du processus) et la borne à droite est ouverte (indiquant l'inaccomplissement du processus), d'où l'opérateur 'PROC ${ }_{\mathrm{J}}$ ' appliqué sur elle. De ce qui précède, on déduit que : un état n'est caractérisé ni par une borne de début, ni par une borne de fin, ces deux bornes n'appartiennent pas à l'intervalle d'actualisation de l'état ; un événement possède nécessairement un premier instant (un début) et un dernier instant (un terme) d'actualisation ; un processus est caractérisé par un premier instant (un début) et par une absence de dernier instant d'actualisation en indiquant ainsi son inaccomplissement.

Des diagrammes permettent de visualiser dans un espace à deux dimensions, comme dans la géométrie analytique de René Descartes, les différences profondes entre les aspects de base. Sur l'axe vertical sont projetées les différentes valeurs instantanées d'une variation d'un changement ; sur l'axe horizontal viennent s'inscrire les intervalles temporels (ouverts, fermés, semi-ouverts) d'actualisation des états, des événements et des processus (figure 2).
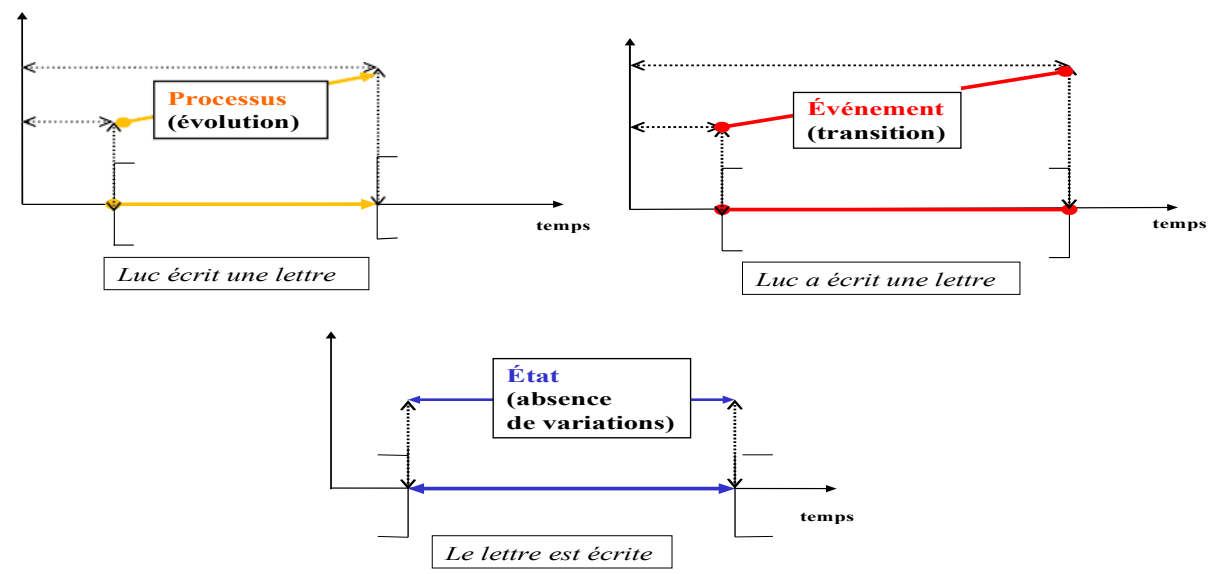

Figure 2 : Diagrammes des états / processus / événements

Donnons quelques exemples. L'énoncé Luc est sage renvoie à un état qui est généralement borné car, par cette énonciation, on ne dit pas que Luc a été ou sera toujours sage. L'énoncé Cet après-midi, Luc a été sage exprime que l'événement 'Luc a été sage' a eu une occurrence durant l'intervalle temporel déterminé par cet après-midi. L'énoncé Luc devient sage exprime un processus en cours qui est par conséquent inaccompli.

Un état est très souvent borné avec une durée pouvant être mesurée par la distance entre ses deux bornes ouvertes, mais un état peut être parfois non borné (dans ce cas, ses bornes ouvertes sont rejetées aux extrémités du référentiel) ; on obtient ainsi une opposition entre état contingent (Actuellement, Luc est vraiment handicapé), état permanent (Luc est handicapé depuis sa naissance) et état non borné d'une vérité générale (La somme des angles d'un triangle est égale à deux droits). Un processus, tout comme un état, ne sont jamais réductibles à un instant ponctuel. L'occurrence d'un événement est généralement non ponctuelle et possède dans ce cas une durée interne (Luc a travaillé tout l'après-midi). Un événement peut être ponctuel, d'où l'impossibilité de *A cet instant, Luc a sursauté durant une heure (que l'on peut comparer à A cet instant, Luc a sursauté puis, aussitôt, il a repris son air serein) ou être compatible avec une durée comme dans L'éclair provoqué par la bombe atomique a brillé pendant quelques secondes ou dans La bombe atomique a éclaté durant plusieurs secondes, formant un champignon.

Etant directement liés à une forme de perception interprétative des mouvements et des changements, les concepts aspectuels de base (état, événement et processus) sont de nature cognitive. Ils sont appréhendés par des opérateurs qui actualisent une relation prédicative 
sur des intervalles topologiques qualitatifs qui ne nécessitent pas d'indications métriques supplémentaires et entretiennent entre eux des rapports dialectiques (au sens de Bachelard). En effet, un état contingent, comme il est actuellement malade, est borné par deux événements contigus à cet état : un premier événement a fait entrer le sujet dans l'état (il est tombé malade), un second l'en fera sortir (il guérira bientôt). Un événement, comme il est tombé malade, est nécessairement borné par deux états contigus, un état antérieur (il n'est pas malade) et un état postérieur (il est malade). Un processus, comme il est en train de tomber malade, implique l'existence d'un état antérieur (il n'est pas malade) à partir duquel se développe une succession de changements de propriétés (d'où un nécessaire premier instant de changement), sans que soit toujours indiquée une orientation vers un état explicitement visé et considéré comme télique. Par exemple, Luc nage dans la rivière est un processus inaccompli atélique (aucun terme n'est visé), tandis que Luc nage vers la rive opposée est un processus inaccompli télique puisqu'un but (l'atteinte de la rive opposée) est indiqué. ${ }^{\text {iv }}$ Un processus inaccompli atélique comme Luc boit / Luc lit un journal, ou télique comme Luc boit une coupe de champagne / Luc lit d'un bout à l'autre le journal, devient un accompli lorsque la borne de droite se ferme (avec indication d'un dernier instant) et, dans ce cas, le processus devient un processus accompli qui engendre un événement, ce dernier est ou bien un simple événement comme dans Luc a bu / Luc a lu un livre toute la matinée, ou bien un événement complet (ou achevé) ne peut plus être prolongé au-delà du terme final, comme dans Luc a bu toute la bouteille de champagne / Luc a lu le livre dans la journée. Nous obtenons ainsi une opposition entre les valeurs aspectuelles 'accompli' et 'achevé', ce que B. Comrie (1996) exprime, en anglais, par l'opposition complete / completed.

Le concept de borne continue acquiert tout son intérêt théorique lorsqu'il s'agit d'expliquer la construction d'état résultatif. En effet, lorsque la borne ouverte d'inaccomplissement d'un processus se ferme, le processus accompli devient un événement et, dans ce cas, la borne fermée qui marque l'accomplissement, est une coupure continue introduite dans un parcours temporel des situations successives et contiguës ; étant continue, cette coupure engendre d'un côté, un événement actualisé sur un intervalle fermé avec un dernier instant et, d'un autre côté, une absence de premier instant à la droite de l'intervalle d'actualisation de l'état engendré par l'accomplissement du processus. En français, le temps grammatical du Passé composé, comme a travaillé, est polysémique : selon les occurrences de cette forme grammaticale dans des contextes précis, le Passé Composé exprime ou bien un événement (Luc a travaillé sur sa thèse pendant cinq heures puis, ensuite, il est allé se promener), ou bien un état résultatif (Luc a travaillé sur sa thèse pendant cinq heures consécutives, il est maintenant fatigué). Dans de nombreuses langues (par exemple en grec, en bulgare et en albanais), ces deux valeurs du Passé composé français sont exprimées par des formes grammaticales différentes, le Parfait et l'Aoriste. L'opposition aspectuelle 'accompli/achevé' acquiert toute sa pertinence théorique et descriptive lorsque l'on compare les deux constructions (a) Luc a peint le mur pendant une heure et (b) Luc a peint le mur en une heure puisque dans (b), l'achèvement de l'écriture de l'article est clairement signifiée, ce qui n'est pas le cas de (a) : l'événement est achevé engendrant un état final dans (b) alors qu'il est simplement accompli dans (a). ${ }^{\mathrm{v}}$

L'Imparfait français possède un large éventail de valeurs et d'emplois dont l'examen systématique permet cependant de dégager une valeur invariante : un procès (état ou processus) actualisé sur un intervalle qui possède nécessairement une borne ouverte à droite ; un énoncé à l'Imparfait ne renvoie donc jamais à un événement bien que certains de ses emplois sembleraient être des exceptions. Prenons, par exemple, le train déraillait. Son occurrence dans (a) Alors que le train déraillait, le conducteur, exténué par sa longue journée de conduite, dormait, renvoie à un processus ; dans ce cas, le Passé composé ou le Passé simple ne peuvent pas venir se substituer à l'Imparfait: *Alors que le train dérailla, le conducteur (...). Dans un tout autre contexte, comme (b) Le conducteur s'endormit et, cinq minutes plus tard, le train déraillait..., la forme de l'Imparfait (appelée imparfait « d'ouverture » ou « de fermeture » ou encore de « de rupture ») vi renvoie non pas à un événement mais à, ce que nous avons appelé, un 'nouvel état' (de l'univers narratif) puisqu'on constate une très nette différence sémantique et d'emploi discursif entre l'énoncé (b) et l'énonciation de (c) Le conducteur s'endormit. Cinq minutes plus tard, le train dérailla. qui exprime ici une succession d'événements d'une narration. Dans une énonciation comme celle de (b), la forme de l'Imparfait construit un nouvel état global de la narration ; un tel état vient modifier l'état antérieur global de la narration en cours ; c'est la 
raison pour laquelle cette forme verbale ne peut occuper que des positions discursives bien spécifiques : soit, en tout début d'un récit (Cette année-là, naissait le plus grand philosophe que la France ait déjà connu) pour marquer une différence radicale par rapport à l'état antérieur de la narration, soit en fin de récit (Après cinq ans d'une cour assidue, ils se marièrent enfin, mais, un mois plus tard, ils divorçaient...). . Dans ce genre d'exemples, l'utilisation de l'Imparfait est étroitement liée à l'occurrence d'un événement (parfois inféré du contexte) mais, contrairement à ce qu'indiquerait une manifestation linguistique d'un événement, marquée par un Passé simple ou un Passé composé, l'emploi discursif de l'Imparfait oriente l'attention et l'interprétation vers l'actualisation d'un nouvel état qui devient directement la conséquence de l'occurrence d'un événement antérieur (parfois implicite), valeur qu'il faut distinguer d'un état résultatif.

La notion d'activité (ou activity) que l'on rencontre souvent dans la littérature relative à l'aspectualité est souvent très confuse. En effet, la notion d'activité ne remplace pas le concept de processus, car il est utile de pouvoir distinguer explicitement l'état d'activité (Luc est au travail) du processus sous-jacent (Luc travaille ou Luc est en train de travailler), les deux intervalles d'actualisation ne coïncidant pas, le premier (un intervalle ouvert) étant nécessairement inclus dans le second (un intervalle semi ouvert). ${ }^{\text {vii }}$

Remarquons que l'intervalle d'actualisation n'est pas toujours réductible à l'intervalle de validation, défini comme un intervalle où une relation prédicative est présentée comme "vraie » à chaque instant de cet intervalle. En effet, si pour un état, les deux sortes d'intervalles (d'actualisation et de validation) coïncident, l'intervalle d'actualisation d'un événement doit être distingué de son intervalle de validation puisque souvent un événement n'est vrai qu'au dernier instant de son actualisation et non pas à chacun des instants qui précède ce dernier instant. Prenons, par exemple, des événements exprimés par Luc a gagné la course ou Luc a lu entièrement ce roman, ceux-ci sont déclarés vrais uniquement aux derniers instants de leurs actualisations et non pas pendant le cours de leurs actualisations. Quant au processus télique dans Luc est en train d'atteindre le sommet ou Luc est en train de gagner le Marathon de New York, ils s'actualisent sur un intervalle semi-ouvert, emboîté dans un intervalle fermé (l'intervalle qui actualisera éventuellement l'événement achevé et complet) ; ces processus téliques sont présentés comme potentiellement vrais à chaque instant de l'actualisation des processus, à condition toutefois qu'ils aboutissent à leurs termes.

Nous présentons une partie de la carte sémantique des principaux concepts aspectuels (voir Desclés et Guentchéva (2012), Desclés 2016) ; dans cette carte, la flèche pleine représente une relation de généralisation et la flèche en pointillé représente une relation d'association.

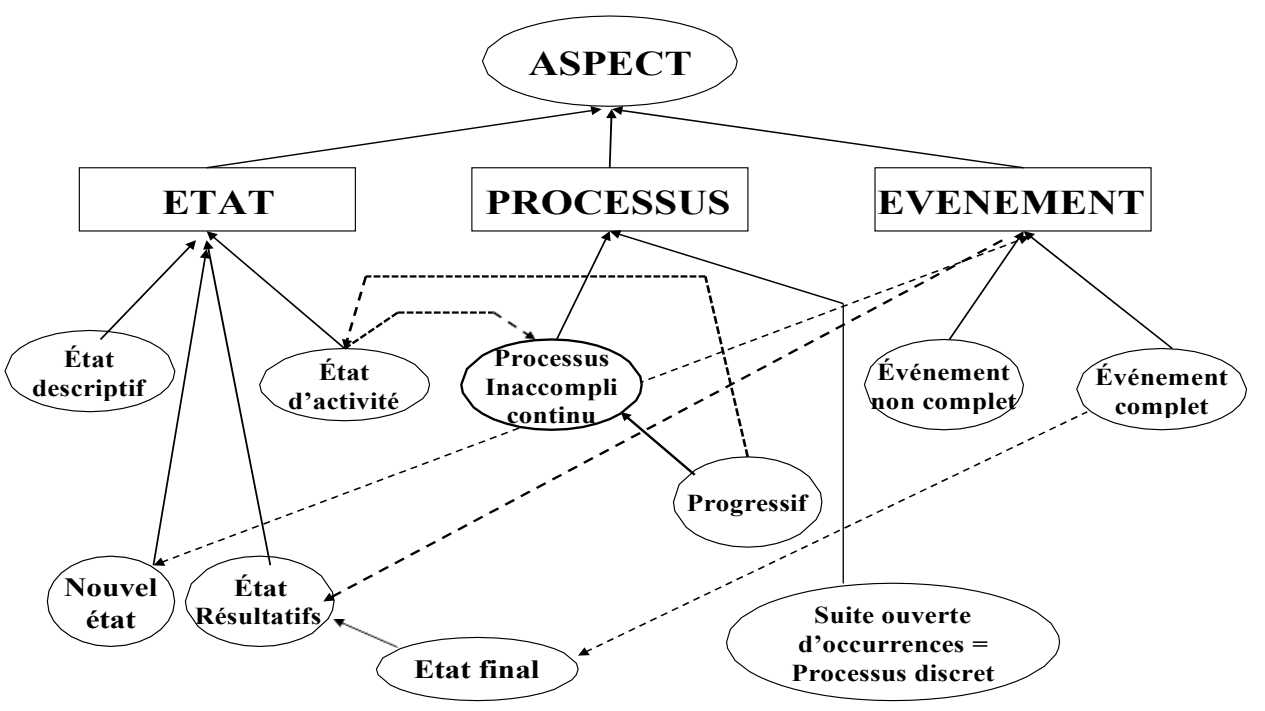

Figure 3 : Carte (simplifiée ) des différents concepts aspectuels

Pour mieux comprendre les définitions des concepts proposés dans le cadre théorique qui est ici défendu, B. Pottier (dans une discussion orale) nous a proposé de traiter 
systématiquement, avec des représentations sous forme de diagrammes, le paradigme d'exemples (obligatoirement contextualisés dans certains cas) engendrés par le schéma de phrase «Christophe Colomb verbe conjugué l'Amérique », avec : viii

verbe conjugué = découvre / a découvert / a eu découvert / découvrit / eut découvert / découvrira / aura découvert / aura eu découvert / découvrait / avait découvert / aurait découvert / va découvrir / allait découvrir / découvrît / eût découvert .

Il serait souhaitable et intéressant que les diverses approches théoriques de l'aspectualité puissent se confronter sur un tel paradigme, ce qui permettrait de déterminer les convergences et les divergences pour mieux discuter les avantages théoriques de telle ou telle représentation métalinguistique.

\section{Enonciation de l'aspectualisation}

Tout énoncé est le résultat d'une énonciation, c'est-à-dire d'un acte de " prise en charge » d'un contenu prédicatif (exprimé par un dictum propositionnel) par un énonciateur (désigné par 'EGO'), au moyen de différentes opérations (dites énonciatives) dont font partie les opérations d'aspectualisation et de repérage temporel (Desclés 2009). Ces opérations de prise en charge énonciative constituent le modus dans la décomposition en modus et dictum de Ch. Bally (1932/1965). Remarquons que l'énonciateur EGO ne doit pas être confondu avec l'occurrence d'un locuteur déterminé par les conditions pragmatiques d'une énonciation particulière ; le signe ' $j e$ ' n'exprime pas toujours une simple identification entre sa référence pragmatique et l'énonciateur EGO, car il peut également renvoyer à une identification avec un « locuteur rapporté » (qui peut être le co-énonciateur TU ou l'absent IL du dipôle dialogique énonciateur-co-énonciateur) d'une énonciation que EGO rapporte directement ou indirectement en prenant éventuellement certaines distances. Dans l'analyse de l'activité de langage, la prise en compte des conditions d'énonciation introduit un opérateur primitif d'énonciation, désigné ici par 'EGO-DIT' ou bien l'opérateur "I am saying ", souvent effaçable car nécessaire dans l'énoncé produit, selon la Grammaire d'opérateurs de Z. Harris (1976).

L'acte d'énonciation ne peut évidemment pas être appréhendé sous la forme d'un événement ponctuel dont l'actualisation serait l'instant $\mathrm{T}^{0}$ (désigné souvent, dans la littérature linguistique, par « le moment d'énonciation »). Dans notre approche, l'acte d'énonciation est conceptualisé comme un processus inaccompli, qui résulte de la composition de l'opérateur minimal d'énonciation 'EGO-DIT' avec l'opérateur aspectuel processuel 'PROC $\mathrm{J}_{0}$ ' qui actualise l'énonciation d'un dictum sur l'intervalle semi-ouvert $\mathrm{J}_{0}$ (l'intervalle d'énonciation) dont la borne droite est ouverte, désignée par ' $\mathrm{d}\left(\mathrm{J}_{0}\right)$ ', et identifiée à l'instant $\mathrm{T}^{0}$, la borne d'inaccomplissement de l'énonciation en cours. Le processus d'énonciation s'applique à son opérande, relation prédicative (un dictum), qui est également déjà aspectualisée.

La relation temporelle complexe entre d'un côté, l'intervalle topologique d'actualisation de la relation prédicative aspectualisée et, d'un autre côté, l'intervalle d'énonciation $\mathrm{J}_{0}$, est représentée, de façon économique par l'activité de langage, uniquement par une relation temporelle qui s'établit entre certaines bornes de ces intervalles; cette relation entre bornes (par exemple, les bornes droites des intervalles) est ensuite composée avec les opérateurs aspectuels afin de construire un opérateur aspectuo-temporel complexe qui a pour trace linguistique un morphème grammatical (simple ou composé) accompagné souvent d'autres marqueurs comme des préverbes, des prépositions et des adverbes. ${ }^{i x}$ Désignons par ' $\lambda$ ' ce que A. Culioli $(1968,1999)$ appelle « lexis », un analogue d'un dictum (c'est-à-dire ce qui est à dire mais pas encore dit car non encore pris en charge par un énonciateur). Le symbole ' $\lambda$ ' désigne une relation prédicative propositionnelle quelconque non encore aspectualisée et non encore localisée dans la temporalité du référentiel temporel de l'énonciateur (ou dans un autre référentiel temporel). Le symbole ' $\mathrm{ASP}_{\mathrm{I}}$ ' désigne un opérateur générique d'aspectualisation qui actualise, sur un intervalle ' $I$ ', une lexis ' $\lambda$ ' ; les propriétés topologiques de l'intervalle 'I' sont spécifiées par le choix d'une aspectualisation (entre autres, par les opérateurs $\mathrm{ETAT}_{\mathrm{O}}, \mathrm{EVEN}_{\mathrm{F}}$ et $\mathrm{PROC}_{\mathrm{J}}$ ). Le schéma de relation temporelle $\left[\mathrm{d}(\mathrm{I}) \operatorname{Rep} \mathrm{d}\left(\mathrm{J}_{0}\right)\right]$ entre les bornes droites des intervalles ' $\mathrm{I}$ ' et ' $\mathrm{J}_{0}$ ' signifie que la borne droite 'd(I)' est repérée (désignée par 'Rep'), par une valeur de concomitance $(=)$ ou d'antériorité 
$(<)$ ou de postériorité $(>)$, par rapport à la borne droite $\mathrm{d}\left(\mathrm{J}_{0}\right)$; cette dernière est identifiée à l'instant $\mathrm{T}^{0}$ qui est un repère fixe dans le référentiel énonciatif 'REN' créé par l'énonciation. En désignant par ' $\&$ ' la conjonction de deux expressions, le schème minimal d'énonciation prend la forme applicative exprimée par des applications d'opérateurs à leurs opérandes :

$$
\text { (I) } \begin{aligned}
& \left(\text { PROC }_{\mathrm{J} 0} \text { o EGO-DIT }\right)\left(\&\left(\operatorname{ASP}_{\mathrm{I}}(\lambda)\right)\left(\left[\mathrm{d}(\mathrm{I}) \operatorname{Rep} \mathrm{d}\left(\mathrm{J}_{0}\right)\right]\right)\right) \\
& =\operatorname{PROC}_{\mathrm{J} 0}\left(\text { EGO-DIT }\left(\&\left(\operatorname{ASP}_{\mathrm{I}}(\lambda)\right)\left(\left[\mathrm{d}(\mathrm{I}) \operatorname{Rep} \mathrm{d}\left(\mathrm{J}_{0}\right)\right]\right)\right)\right)
\end{aligned}
$$

Ce schème signifie : « l'énonciateur EGO est en train de prendre en charge sur l'intervalle d'énonciation $\mathrm{J}_{0}$ une relation prédicative aspectualisée 'ASP $\mathrm{A}_{\mathrm{I}}(\lambda)$ ', conjointement avec son repérage temporel $\left[\mathrm{d}(\mathrm{I}) \operatorname{Rep} \mathrm{d}\left(\mathrm{J}_{0}\right)\right.$ ], qui spécifie sa coordonnée temporelle dans le référentiel énonciatif de l'énonciateur EGO ». Les spécifications de l'opérateur aspectuel $\mathrm{ASP}_{\mathrm{I}}$ sont soit l'opérateur statif $\mathrm{ETAT}_{\mathrm{O}}$ actualisé sur un intervalle ouvert $\mathrm{O}$, soit l'opérateur événementiel $\mathrm{EVEN}_{\mathrm{F}}$ actualisé sur un intervalle fermé $\mathrm{F}$, soit l'opérateur processuel $\mathrm{PROC}_{\mathrm{J}}$ actualisé sur un intervalle semi-ouvert $\mathrm{J}$. Nous en avons vu précédemment un certain nombre d'exemples qui étaient tous relatifs au référentiel énonciatif.

Le schème précédent (I) doit être complexifié pour tenir compte des conditions aspectuelles qui dépendent : (i) uniquement de la signification intrinsèque du prédicat verbal (prédicats statiques, prédicats évolutifs aussi bien cinématiques que dynamiques, prédicats atéliques ou téliques) ; (ii) de la modification partielle ou complète d'un actant du prédicat verbal ; (iii) des conditions aspectuelles globales portant sur toute la relation prédicative et (iv) des changements de référentiels (voir ci-dessous). Nous ne présentons pas ici la forme symbolique de ce schème complexifié.

\section{Différents référentiels}

Dans la prise en charge énonciative d'une relation prédicative, l'énonciateur peut chercher à indiquer son attitude par rapport à la vérité de ce qu'il énonce (Je dis qu'il est vrai que...) en faisant ainsi une assertion qui l'engage complètement ce qui n'est pas le cas d'une simple déclaration modulable (Je dis que...). ${ }^{\mathrm{x}}$ L'énonciateur peut également indiquer, par son énonciation, une forme d'incertitude relative à son propos par le biais d'une modalité épistémique (Je dis qu'il est plus ou moins certain / possible / plus ou moins probable...) ou indiquer une modalité appréciative (Je dis qu'il est juste /heureux /malheureux...) ou encore une croyance ou un souhait (Je dis que je suis persuadé /que je crois / que j'espère...). L'énonciateur peut également prendre en charge le rapport d'une énonciation d'un autre locuteur, en reproduisant, plus ou moins fidèlement, les propos de ce dernier. Il peut également se référer à des connaissances attribuées à un tiers, en s'en dissociant (Selon Aristote, la terre serait le centre $d u$ monde) ou en y adhérant complètement (Depuis Copernic et Galilée, la terre n'est plus le centre du monde). Ces différentes opérations de prise en charge énonciative se composent ${ }^{\mathrm{xi}}$, dans la représentation métalinguistique, avec l'opérateur aspectualisé d'énonciation ' $\mathrm{PROC}_{\mathrm{J} 0} \mathbf{0}$ EGO-DIT', pour constituer un opérateur plus complexe, appelé modus qui s'applique à un dictum ou, en d'autres termes, à une lexis. Les opérations de prise en charge de l'énonciateur EGO d'un contenu prédicatif laissent des traces linguistiques dans l'énoncé produit et ce sont ces traces qui permettent aux linguistes de reconstruire les opérations énonciatives du modus.

Le schème minimal énonciatif (I) trouve son actualisation dans le référentiel énonciatif temporel, désigné par REN, et engendré par l'acte de parole de l'énonciateur EGO, en interaction dialogique avec son co-énonciateur TU. Ce référentiel temporel est une organisation continue d'instants ordonnés à partir du processus énonciatif dont la borne droite $\mathrm{T}^{0}$ (le repère temporel d'inaccomplissement de l'énonciation en cours). Rappelons que l'instant $\mathrm{T}^{0}$ constitue un repère fixe dans le référentiel énonciatif temporel. Ce dernier n'est pas représentable sous la forme d'une ligne continue totalement ordonnée et orientée puisque les situations, dont l'actualisation est simplement visée à partir de l'acte énonciatif comme étant à venir, n'ont pas le même degré de certitude (étant quasi-certaines, plus ou moins probables, possibles, nécessaires, voire impossibles...) que les situations déjà actualisées dans «le passé » de l'énonciateur. Sur le concept de référentiel temporel, on peut se reporter à Desclés (1995), ainsi qu’à Desclés et Guentchéva (2010/2012). 
Dans une analyse énonciative des énoncés et discours, il est indispensable de distinguer le référentiel énonciatif du référentiel externe (REX) puisque parler, c'est-créer un référentiel énonciatif autonome détaché du (ou en rupture avec le) référentiel externe des instants d'un temps cosmique, calendaire ou des annales, et d'une temporalité physique externe. Alors que l'instant $\mathrm{T}^{0}$ est un repère fixe dans le référentiel énonciatif, sa projection $t_{0}$ dans le référentiel externe est un repère mobile qui avance avec le « flux » du temps (time). La relation de rupture (ou de déconnexion) entre les deux instants $\mathrm{T}^{0}$ et $\mathrm{t}_{0}$, notée $\left[\mathrm{T}^{0}\right.$ $\# \mathrm{t}_{0}$ ], établit un changement de référentiels entre le REN et le REX. Néanmoins, il arrive que, dans certaines énonciations particulières, ces deux référentiels puissent être localement synchronisés ; en effet, l'énonciateur peut vouloir synchroniser son énonciation avec une période externe (exemple : aujourd'hui, 26 mars 2020) ou encore, dans un reportage, synchroniser complètement son énonciation avec la succession des événements externes qu'il verbalise sous la forme de processus encore inaccomplis au moment où ils sont énoncés (Zidane s'empare du ballon, ajuste son tir et marque un but...).

A côté du référentiel énonciatif organisé par l'énonciation, d'autres référentiels temporels doivent être également pris en considération dans l'analyse des discours et des textes. En effet, les différents procès verbalisés d'une narration ou d'une fiction ne peuvent pas être localisés dans le référentiel énonciatif temporel et sont organisés temporellement dans un autre référentiel, le référentiel non actualisé, noté RNA, où chaque état, événement ou processus inaccompli est temporellement repéré (par concomitance ou par antériorité / postériorité) par rapport à d'autres états, événements ou processus inaccomplis de ce même référentiel et non pas par rapport au processus d'énonciation ou à toute autre localisation dans le référentiel énonciatif. Les procès dans une narration relèvent, selon E. Benveniste 1966), du " plan de l'histoire » opposé au «plan d'énonciation » car ils ne sont pas en général temporellement reliés directement à leurs énonciations ; des marqueurs discursifs comme Un jour / Ce jour-là / Il était une fois / Cette histoire a commencé lorsque..., sont les indications d'une " relation de rupture » (ou de déconnexion) entre le RNA, qui a une organisation temporelle narrative autonome, et le REN. Là encore, l'énonciateur-narrateur peut synchroniser sa narration avec des procès du REN, en présentant les événements localisés dans le RNA sous la forme de processus en train de s'accomplir « devant les yeux du co-énonciateur » dans le REN, d'où, dans de nombreuses langues, l'emploi du temps Présent, dit de narration. Certaines propositions générales, comme $2+2$ font 4 ou Il n'y a pas de fumée sans feu, dénotent des situations toujours vraies ou présentées comme telles et, de ce fait, représentés dans les langues par des procès atemporels et localisés et localisés dans un référentiel des vérités générales. Dans le cas de l'utilisation discursive de proverbes, ces procès viennent se synchroniser et s'actualiser avec des procès analogues localisés dans le référentiel énonciatif.

L'activité discursive revient souvent à envisager une possibilité d'actualisation d'un procès hypothétique avec des conséquences certaines, probables ou simplement éventuelles et donc à poser des relations temporelles et modales dans un référentiel hypothétique, puis à verbaliser l'actualisation ou l'absence d'actualisation de ces procès dans le référentiel énonciatif. On en déduit, en fonction des procès actualisables ou non actualisés dans le référentiel énonciatif, les valeurs modales, par exemple, l'expression d'une relation contrefactuelle passée (Si j'avais eu de l'argent, je t'en aurais donné), ou l'expression d'une éventualité actuelle (Si j'ai de l'argent sur mon compte, je te donne la somme que tu me réclames), ou encore l'expression d'une éventualité ultérieure ( $\mathrm{Si}$, l'an prochain, j'obtiens mon poste, je t'en ferai bénéficier). Des propos tenus par un locuteur, autre que l'énonciateur, peuvent être rapportés directement par l'énonciateur sans que ces propos soient entièrement « pris en charge » par l'énonciateur. Pour cela, l'énonciateur fait appel à un autre référentiel (le référentiel des discours rapportés), en accompagnant souvent ce qui est rapporté d'un jugement évaluatif (exemple : X a dit en riant / a déclaré officiellement / a affirmé... ; Xa selon moi, menti en disant : « ...»).

L'acte de négation d'un énonciateur (comme Paul n'est pas là) implique qu'un procès affirmatif (Paul est là) soit représenté dans un autre référentiel avec l'indication qu'il n'est pas actualisé dans le référentiel énonciatif.

\section{Conclusion}

Dans une approche théorisée des représentations interprétatives de l'aspectualité, de la temporalité et de la modalité, formalisées à l'aide de la topologie générale, il semble 
difficile de pouvoir se passer du concept de changement de référentiels temporels. Il est vrai que des linguistes comme Benveniste (1966), Seiler (1952), Ivanchev (1971), Weinrich (1972), Culioli (1980), Fleischman (1990), Banfield (1995) utilisent des oppositions notionnelles comme discours / histoire, énonciation / narration, actuel / inactuel... sans toujours en tirer toutes les conséquences qui contribuent à cerner la complexité de l'activité de langage manifestée par les langues. Chaque langue grammaticalise de différente façon les compositions entre opérateurs aspectuels, relations temporelles et opérateurs modaux avec l'indication de relations entre référentiels temporels (par mise en rupture et par synchronisation éventuelle).

Du point de vue épistémologique, il est intéressant de souligner que, dans une discipline scientifique, une théorisation imagine des divisions conceptuelles de plus en plus fines mais parfois ad hoc au fil des exemples rencontrés, ou adopte des conceptualisations trop générales et de ce fait peu opératoires ; parfois, une théorisation arrive à progresser plus radicalement, en particulier lorsqu'elle a réussi à mathématiser un réseau de concepts de base, c'est-à-dire les concepts théoriques les plus spécifiques, construits à partir d'un ensemble réduit d'opérations définies avec précision. C'est le cas de la prise en compte de la nature topologique des différents types de bornes d'un intervalle d'actualisation d'une relation prédicative aspectualisée, ainsi que du concept de borne continue ; cela nous conduit à donner des significations et des représentations symboliques accompagnées de diagrammes interprétatifs du réseau des concepts aspectuels, en faisant mieux apparaître leurs liens et leurs oppositions leurs oppositions :

état contingent (borné) /non contingent (non borné) ; accompli/inaccompli ; accompli/achevé ; perfectivité (avec signification d'un achèvement) / imperfectivité (avec absence d'une signification explicite d'un achèvement) ; processus télique (avec indication d'un but visé) /atélique (sans indication d'un but visé) ; états résultatifs (l'état résultant, l'état passif, l'état conséquent, l'état d'expérience...) d'une occurrence d'un événement ; processus continu/processus discret; habitude analysée comme une itération discrète (énumérable) d'événements ou de processus ; état d'activité / processus discret d'occurrences d'événements...

Le manque de place nous empêche de donner pour chacun de ces concepts des définitions, accompagnées de diagrammes interprétatifs.

\section{Références bibliographiques}

Bally, Ch. 1932/1965. Linguistique générale et linguistique française. Berne : Franke.

Banfield A. 1995. Phrases sans paroles, Paris : Seuil. Trad. fr. de Unspeakable Sentences : Narration and Representation in the Language of Fiction, 1982. Londres : Routledge \& Kegan Paul.

Benveniste, E., 1959/1966. Les relations de temps dans le verbe français, BSL, LIV, fasc. 1. Repris dans Problèmes de linguistique générale, Vol. I: 237250. Paris: Gallimard.

Comrie, B. 1976. Aspect: An introduction to the study of verbal aspect and related problems. Cambridge : Cambridge University Press.

Culioli A. 1968. La formalisation en linguistique, Cahiers pour l'analyse, 9, Généalogie des Sciences, 106-117, repris dans Culioli (1999: 17- 29).

Culioli, A. 1980. Valeur aspectuelle et opérations énonciatives: l'aoristique, in J. David et R. Martin (eds) : Notion d'aspect, Paris: Klincksieck, 181-193.

Culioli, A. 1999. Pour une linguistique de l'énonciation, Formalisation et opérations de repérage, t. 2, Paris : Ophrys.

Curry, H. B. \& R. Feys, 1958. Combinatory Logic, Vol. I, North-Holland.

Curry, H. B., J. Hindley \& J.P. Seldin, Combinatory Logic, vol II, Amsterdam, North Holland.

Dahl, Ö. 1981. On the Definition of the Telic-Atelic (Bounded - Unbounded) Distinction, in P. J. Tedeschi and A. Zaenen (eds.), Syntax and Semantics, Tense and Aspect, Vol. 14: 79-90. Academic Press, New York.

Declerck, R. 2007. Distinguishing between the aspectual categories '(a)telic', '(im)perfective' and '(non)bounded', Kansas Working Papers in Linguistics, Vol. 29 (2007), 48-64. (URL: http://hdl.handle.net/1808/1787

Dhombres, J. 1978. Nombre, mesure et continu. Epistémologie et histoire. Cedic/Fernand Nathan.

Depraetere, I. 1995. On the Necessity of Distinguishing between (un)boundedncss and (a)telicity, Linguistics and Philosophy 9, 37-61.

Dedekind, R., 1893. Was sind und was sollen die Zahlen? Trad. de H. Sinaceur dans La création des nombres, . coll2008 « Mathesis », Paris : Vrin. 
Desclés, J.-P. 1980. Construction formelle de la catégorie grammaticale de l'aspect (essai). In J. David and R. Martin (eds.), La notion d'aspect, 198-237. Paris : Klincksieck.

Desclés, J.-P. 1989. State, event, process and topology. General Linguistics, 293: 159-200.

Desclés, J.-P. 1995. Les référentiels temporels pour le temps linguistique, Modèles linguistiques, XVI, $2: 9-36$.

Desclés, J-P., 2009. Opérations de prise en charge et d'engagement : une carte sémantique des catégorisations opérées par les langues, Langue française, 162, 29-53,.

Desclés, J-P., 2016. A cognitive and conceptual approach to tense and aspect markers. In Z. Guentchéva (ed.) Aspectuality and Temporality. Descriptive and theoretical issues, 27-60. Amsterdam/ Philadelphia: John Benjamins Publishing Company.

Desclés, J.-P. \& Z. Guentchéva, 1995. Is the notion of process necessary? In P. M. Bertinetto, V. Bianchi, Ö. Dahl, and M. Squartini (eds.), Temporal reference, aspect, and actionality. Vol. $1: 55-70$. Turin: Rosenberg and Sellier.

Desclés J.-P. et Guentchéva Z., 2010/2011. Référentiels aspecto-temporels : une approche formelle et cognitive appliquée au français, Congrès Mondial de Linguistique Française - CMLF 2010, in Neveu F., V. Muni Toke, J. Durand, T. Klingler, L. Mondada, S. Prévost (éds), Institut de Linguistique Française, Paris, 1675-1696. Repris dans Bulletin de la Société de Linguistique, 2011, 106 (1) : 95-127.

Desclés, J.-P. \& Z. Guentchéva, 2012. Universals and Typology. In R. Binnick (ed), The Oxford Handbook of Tense and Aspect, chap. 4, 124-154. Oxford: Oxford University Press.

Desclés J.-P., G. Guibert, B. Sauzay, 2016. Logique combinatoire et $\lambda$-calcul : des logiques d'opérateurs, Vol. I ; Calcul des significations par une logique d'opérateurs, Vol. II. Toulouse : Cépaduès.

Fleischman, S., 1990. Tense and Narrativity. Austin : University of Texas Press.

Gosselin, L. 1996. Sémantique de la temporalité en français (Un modèle calculatoire et cognitif du temps et de l'aspect), Duculot.

Gosselin, L. 2010. Les modalités en français : La validation des représentations. Amsterdam/New York : Rodopi.

Guentchéva, Z., 1990. Temps et aspect. L'exemple du bulgare contemporain. Paris : Éditions du Centre national de la recherche scientifique.

Guentchéva, Z., 1995. L'imparfait perfectif en bulgare. Modèles Linguistiques, 16(2): 73-94.

Harris, Z. 1976. Notes du cours de syntaxe, Paris : Seuil.

Ivanchev, Sv., 1971. Problemi na aspektualnostta v slavjanskite ezici. Sofia: Bălgarska Akademija na Naukite.

Kozlowska, M. 1997. Bornage et ordre temporel. Cahiers de linguistique française, 19 : 345-368.

Landaburu, J, 2007. La modalisation du savoir en langue andoke (Amazonie colombienne). In Z. Guentchéva \& J. Landaburu (Eds.), L'énonciation médiatisée II: Le traitement épistémologique de l'information; Illustrations amérindiennes et caucasiennes, 23-47. Leuven : Peeters.

Lyons, J. 1977. Semantics. Cambridge: Cambridge University Press.

Mourelatos, A.P.D. (1981), Events, Processes, and Slatcs. Syntax and Semantics 14. New York. Academic Press, 191-212.

Sasse, H.-J. 2002. Recent activity in the theory of aspect: Accomplishments, achievements, or just non progressive state ? Arbeitspapiere no. 40. Köln: Institut für Sprachwissenschaft, Universität zu Köln. Published in Linguistic Typology, 6, 199-271.

Reichenbach, H, 1947. Elements of Symbolic Logic.New York: MacMillan.

Seiler, H.-J., 1952. L'aspect et le temps dans le verbe neo-grec. Paris : Les Belles Lettres.

Tasmowski-De Ryck, 1985. L'imparfait avec et sans rupture. Langue française, $\mathrm{n}^{\circ} 67:$ 59-77.

Vendler, Z., 1957. Verbs and Times. The Philosophical Review, 66 (2): 143-160. Repris dans Linguistics in Philosophy, Ithaca: Cornell University Press, 97-121.

Weinrich, H. 1973. Le temps (trad. M. Cost). Paris : Seuil.

\footnotetext{
Sur la continuité, voir, par exemple, J. Dhombres(1978).

${ }^{\text {ii }}$ Vendler (1957) distingue les notions de state, activity, accomplishment et achievement qui, selon nous, comme pour beaucoup d'autres auteurs, ne sont pas définies, étant uniquement appréhendées par des exemples. Dans cette approche, les notions pourtant différentes de processus et d'état d'activité sont rassemblées sous l'étiquette activity et achievement renvoie à un événement uniquement ponctuel.

${ }^{\text {iii }}$ Sur la distinction entre état, événement et processus, voir aussi Desclés (1980, 1989).

${ }^{\text {iv }}$ Sur la distinction entre télique et atélique d'une part et borné et non borné, d'autre part, voir en particulier Depraetere (1995), Koslowska (1996), Declerck (2007).

${ }^{\mathrm{v}}$ La distinction accompli/achevé est grammaticalisée dans de nombreuses langues, notamment dans les langues slaves et en particulier en bulgare où l'on oppose un Aoriste perfectif à un Aoriste imperfectif, ainsi qu'un Imparfait imperfectif à un Imparfait perfectif (Guentchéva 1990, 1995) ${ }^{\text {vi }}$ Voir par exemple L. Tasmowski (1985) et la littérature citée.

${ }^{\text {vii }}$ Sur la notion d'état d'activité, voir Desclés et Guentchéva (1995).
} 


\footnotetext{
viii La réponse que nous avons transmise à B. Pottier fera l'objet d'une publication. Le manque de place ne nous permet pas de présenter les analyses accompagnées de leurs diagrammes interprétatifs. ${ }^{\text {ix }}$ La composition s'effectue formellement dans le cadre du formalisme de la Logique Combinatoire typée de H. B. Curry $(1958,1972)$, qui est une logique d'opérateurs quelconques, composables et transformables, de façon intrinsèque, par les opérateurs abstraits, appelés combinateurs, engendrés à partir d'un nombre réduit de combinateurs élémentaires ; voir aussi Desclés, Guibert et Sauzay (2016).

${ }^{\mathrm{x}}$ La notion d'engagement est grammaticalisée dans certaines langues comme, par exemple en andoke, langue amérindienne parlée en Colombie (Landaburu 2007).

${ }^{x i}$ Les compositions de ces différents opérateurs constitutifs du modus sont effectuées toujours dans le formalisme de la Logique Combinatoire.
} 